\title{
Development of the neutron polarizer for the T-violation search using compound nuclei
}

\author{
T. Okudaira ${ }^{1}$, T. Oku ${ }^{1}$, K. Sakai ${ }^{1}$, T. Ino ${ }^{2}$, H. Hayashida ${ }^{3}$, K. Hiroi $^{1}$, T. Shinohara ${ }^{1}$, K. \\ Kakurai $^{3}$, K. Aizawa ${ }^{1}$, H. M. Shimizu ${ }^{4}$, M. Kitaguchi ${ }^{4}$, T. Yamamoto ${ }^{4}$ \\ ${ }^{1}$ Japan Atomic Energy Agency, Japan \\ ${ }^{2}$ High Energy Accelerator Research Organization, Japan \\ ${ }^{3}$ Comprehensive Research Organization for Science and Society, Japan \\ ${ }^{4}$ Nagoya University, Japan \\ E-mail: okudairadpost.j-parc.jp
}

\begin{abstract}
The fundamental parity violation is enhanced by $10^{6}$ times at maximum in several neutron induced compound nuclei. There is a theoretical prediction that fundamental T-violating effects can be also largely enhanced in compound nuclei implying that T-violation can be searched for by making very sensitive measurements. A T-violation search experiment using compound nuclei is planned at J-PARC. Polarized epithermal neutron beam, polarized nuclear target, and high count rate neutron detector are necessary for the experiment, and therefore a development of the neutron polarizer using polarized ${ }^{3} \mathrm{He}$ is ongoing at J-PARC in JAEA. Recently, a clean vacuum system to fabricate ${ }^{3} \mathrm{He}$ spin filters was constructed, and several ${ }^{3} \mathrm{He}$ spin filters were fabricated. The current status of the development of the ${ }^{3} \mathrm{He}$ spin filter was reported.
\end{abstract}

The 4th KMI International Symposium (KMI2019)

18-20, February 2019

Nagoya, Japan

*Speaker. 


\section{T-violation search using compound nuclei}

Weak interaction violates parity $(\mathrm{P})$ symmetry in nuclear reactions. Small P-violation with a size of $10^{-7}$ caused by hadronic weak interaction has been observed in the scattering experiment between a polarized proton beam and an unpolarized proton target [U, 口, []]. Very large P-violation with the size of $10^{-1}$ at maximum has been observed in neutron induced compound nuclei such as ${ }^{139} \mathrm{La},{ }^{131} \mathrm{Xe},{ }^{81} \mathrm{Br}$, and so on. The large P-violation occurs at a $\mathrm{p}$-wave resonance located at a tail of large s-wave resonances, and this implies that the fundamental P-violation is largely enhanced by $10^{6}$ times at maximum in the compound nuclei. The enhancement is theoretically explained as a result of an interference between s-wave and p-wave amplitudes. There is a theoretical prediction that the enhancement mechanism can be applicable for time (T) reversal violation, and the fundamental T-violation can be also enhanced in the nuclei whose P-violation is enhanced [⿴囗才]. We are aiming to search for T-violation beyond the Standard Model sensitively using the compound nuclei at J-PARC, and several experiments and developments for the T-violation search are in progress.

The forward scattering cross section of polarized neutrons to polarized nuclei can be represented as

$$
f=A^{\prime}+B^{\prime} \boldsymbol{\sigma} \cdot \hat{\boldsymbol{I}}+C^{\prime} \boldsymbol{\sigma} \cdot \hat{\boldsymbol{k}}+D^{\prime} \boldsymbol{\sigma} \cdot(\hat{\boldsymbol{I}} \times \hat{\boldsymbol{k}})
$$

where $\boldsymbol{\sigma}, \hat{\boldsymbol{I}}$ and $\hat{\boldsymbol{k}}$ are the spin of incident neutron, the spin of the target nucleus and the momentum of the neutron, respectively. Here we assume that the polarization of the target nucleus is pure vector polarization. $A^{\prime}$ is the spin independent (P-even, T-even) term corresponding to the ordinal cross section. $B^{\prime}$ is the spin dependent (P-even, T-even) term corresponding to a neutron spin rotation through the polarized target called a "pseudo-magnetic effect". $C^{\prime}$ is the P-violating (Podd, T-even) term, which is enhanced for several compound nuclei discussed previously. $D^{\prime}$ is the T-violating (P-odd, T-odd) term. Therefore, in order to search for the T-violating cross section, a polarized neutron beam and a polarized nuclear target are necessary.

The T-violating cross section $\Delta \sigma_{\mathrm{T}}$ corresponding to $D^{\prime}$ can written as

$$
\Delta \sigma_{\mathrm{T}}=\kappa(J) \frac{W_{\mathrm{T}}}{W} \Delta \sigma_{\mathrm{P}}
$$

where $\Delta \sigma_{\mathrm{P}}$ is the $\mathrm{P}$-violating cross section, $W_{\mathrm{T}}$ and $W$ are the T-violating and $\mathrm{P}$-violating matrix elements, and $\kappa(J)$ is a function of the spin of a compound nucleus and a partial neutron width of the p-wave resonance, referred to as spin factor [四]. Here, since $\Delta \sigma_{\mathrm{P}}$ is largely enhanced in several compound nuclei, $\Delta \sigma_{\mathrm{T}}$ can be also enhanced in the nuclei when $\kappa(J)$ is not small. Therefore the experimental sensitivity strongly depends on values of the P-violation and $\kappa(J)$. The spin factor $\kappa(J)$ can be determined by measuring the angular distribution of the $\gamma$-ray emitted from the p-wave resonance, and has not yet been measured for almost all nuclei. Recently, $\kappa(J)$ for ${ }^{139} \mathrm{La}$, which has very large P-violation with a size of $10^{-1}$ at $0.74 \mathrm{eV}$ p-wave resonance, has been determined in J-PARC, and the value is order of 1 [ [] ]. This imply that fundamental T-violation is also enhanced by the same order as the P-violation in ${ }^{139} \mathrm{La}$. Additionally, ${ }^{139} \mathrm{La}$ has large natural abundance, and the high nuclear polarization of $50 \%$ has been achieved [6]. From these properties, ${ }^{139} \mathrm{La}$ is 
good candidate nuclei, and we are planning the T-violation search using ${ }^{139} \mathrm{La}$. A highly polarized neutron beam at $0.74 \mathrm{eV}$, which is the p-wave resonance energy of ${ }^{139} \mathrm{La}$, is necessary for the Tviolation search, and the development of a neutron polarizer is conducted in J-PARC.

\section{Development of a ${ }^{3} \mathrm{He}$ spin filter}

$\mathrm{A}^{3} \mathrm{He}$ spin filter is a neutron spin polarization device composed of ${ }^{3} \mathrm{He}$ gas and a special glass cell. Since the neutron absorption cross section of ${ }^{3} \mathrm{He}$ has strong spin dependence, the neutron beam is polarized passing through the polarized ${ }^{3} \mathrm{He}$ gas encapsulated to the glass cell. The ${ }^{3} \mathrm{He}$ spin filter can polarize neutron beam for wide energy range, and is suitable to polarize neutrons at p-wave resonance energy of ${ }^{139} \mathrm{La}$. The glassware of ${ }^{3} \mathrm{He}$ spin filter is made from Aluminosilicate glass, referred to as GE180, which does not include boron. A figure of merit (FOM) of the ${ }^{3} \mathrm{He}$ spin filter can be defined as $\mathrm{FOM}=P^{2} T$, where $P$ and $T$ are neutron polarization and neutron transmission, respectively. FOM is a function of an effective ${ }^{3}$ He thickness in the glass cell, which is represented by a product of a gas thickness and a gas pressure. FOM has maximum at $79 \mathrm{~atm} \cdot \mathrm{cm}$, and neutron transmission and neutron polarization are $30 \%$ and $87 \%$ for $0.74 \mathrm{eV}$ neutrons when the ${ }^{3} \mathrm{He}$ polarization is $70 \%$. We are aiming to develop such large ${ }^{3} \mathrm{He}$ spin filter for the T-violation search experiment. The development of ${ }^{3} \mathrm{He}$ spin filter is ongoing at J-PARC in JAEA.

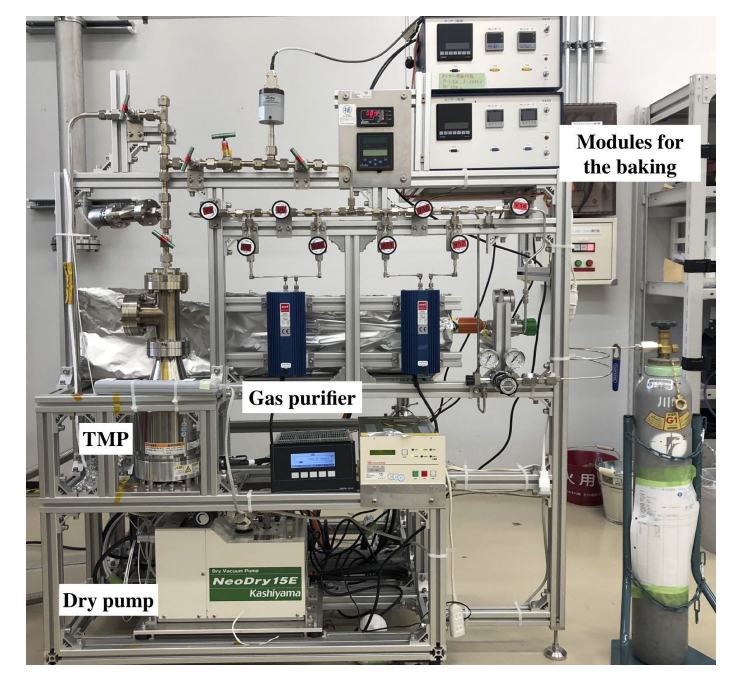

Figure 1: The vacuum system to encapsulate ${ }^{3} \mathrm{He}$ and $\mathrm{Rb}$ to the glass cell.

The isotope ${ }^{3} \mathrm{He}$ is polarized with Spin Exchange Optical Pumping (SEOP) method using alkali metal. The alkali metal is enclosed with ${ }^{3} \mathrm{He}$ gas in the glass cell, and peripheral electrons of the alkali atoms are polarized by the circularly polarized laser. The polarization of the electrons moves to the ${ }^{3} \mathrm{He}$ nuclei with the hyper fine interaction, and the ${ }^{3} \mathrm{He}$ nuclei are polarized. A uniform magnetic field is applied to ${ }^{3} \mathrm{He}$ spin filter using a solenoid and a compensation coil with a magnetic shield to maintain the ${ }^{3} \mathrm{He}$ polarization. The ${ }^{3} \mathrm{He}$ polarization relaxes by the inhomogeneity of the magnetic field, impurities inside the glass cell, collisions with other ${ }^{3} \mathrm{He}$ nucleus, and other effects. 
Especially, a clean vacuum system to encapsulate ${ }^{3} \mathrm{He}$ and alkali metal to the glass cell is important to fabricate ${ }^{3} \mathrm{He}$ which have long relaxation time. A performance of the ${ }^{3} \mathrm{He}$ spin filter can be evaluated from the relaxation time of the ${ }^{3} \mathrm{He}$ polarization. Recently, the vacuum system was constructed at J-PARC as shown in Fig W. Glassware is washed using neutral detergent, pure water, acetone, and ethanol. The glassware is connected to the vacuum system and the glass cell and stainless tube of the vacuum system are baked out over 1 weak at $400{ }^{\circ} \mathrm{C}$ and $200{ }^{\circ} \mathrm{C}$, respectively. The ultimate achievable vacuum is $8 \times 10^{-8} \mathrm{~Pa}$ after the baking. The alkali metal was enclosed to the glass cell after distillations for 2 times. We fabricated two ${ }^{3} \mathrm{He}$ spin filters using $\mathrm{Rb}$ as shown in Fig $\square$ and Fig [ ]. The effective gas thicknesses of the first and second ${ }^{3} \mathrm{He}$ spin filters are measured by a neutron transmission experiment at neutron beamline 10 on the material and life science experimental facility in J-PARC as $4.2 \mathrm{~atm} \cdot \mathrm{cm}$ and $29.3 \mathrm{~atm} \cdot \mathrm{cm}$, respectively.

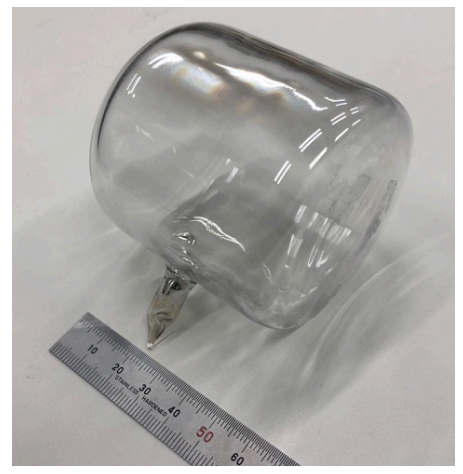

Figure 2: A first ${ }^{3} \mathrm{He}$ spin filter in J-PARC. A diameter and length are $6 \mathrm{~cm}$ and $6.5 \mathrm{~cm}$, respectively.

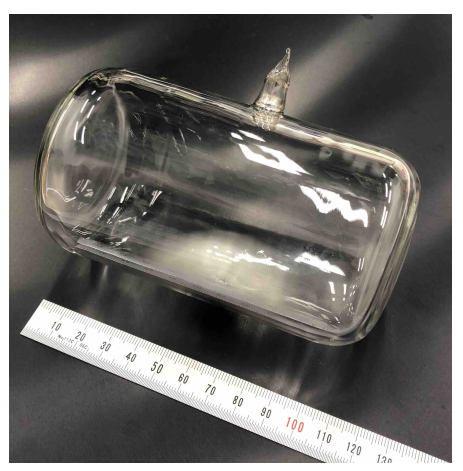

Figure 3: A second ${ }^{3} \mathrm{He}$ spin filter in J-PARC. A diameter and length are $6.5 \mathrm{~cm}$ and $11.5 \mathrm{~cm}$, respectively.

The ${ }^{3} \mathrm{He}$ spin filter is polarized using the laser system with the laser power of $30 \mathrm{~W}$. The wave length of the laser is narrowed at $795 \mathrm{~nm}$, which is an absorption wave length of $\mathrm{Rb}$ atom, using a volume holographic grating. The relative polarization is measured with adiabatic fast passage NMR. We measured the relaxation of the polarization for the first and second ${ }^{3} \mathrm{He}$ spin filters. The relaxation times for the first and second ${ }^{3} \mathrm{He}$ spin filters are 26 hour and 96 hour, respectively. The



Figure 4: The relaxation of the ${ }^{3} \mathrm{He}$ polarization for the second ${ }^{3} \mathrm{He}$ spin filter measured with AFP-NMR. 
short relaxation time of the first ${ }^{3} \mathrm{He}$ spin filter might be caused by an accidental tiny inflow of air in the fabrication process. The relaxation of the ${ }^{3} \mathrm{He}$ polarization for the second ${ }^{3} \mathrm{He}$ spin filter is shown in Fig. 团, and the time dependence of the NMR signal was fitted by exponential function. This relaxation time is enough to perform neutron experiments for a few days, however, longer relaxation time is achievable by improving the fabrication processes, such as the baking and the distillation. After establishing the fabrication process, we will develop the larger ${ }^{3} \mathrm{He}$ spin filters.

\section{References}

[1] J. M. Potter et al., Phys. Rev. Lett. 331307 (1974)

[2] V. Yuan et al., Phys. Rev. Lett. 571680 (1986)

[3] E. G. Adelberger and W. C. Haxton, Ann. Rev. Nucl. Part. Sci. 35501 (1985)

[4] V. P. Gudkov, Phys. Rep. 21277 (1992)

[5] T. Okudaira et al., Phys. Rev. C 97034622 (2018)

[6] P. Hautle and M. Iinuma. NIM A., 440638 (2000) 